\title{
SPREADING ACTIVATION APPLIED IN SEMANTIC NETWORKS OF LANGUAGE NEURAL PROCESSING MODELS
}

\author{
KRATOCHVIL, P[etr]; MACHOVA, S[vetlana] \& KLECKOVA, J[ana]
}

\begin{abstract}
The aim of this article is to find a new experimental text processing model based on mechanisms underlying human brain's neural processing. A considerable possibility of building such model is reexamining of Shank's Conceptual Dependency Theory and Story Understanding. We are offering a new approach to the text processing based on Conceptual Dependency stories and semantic neural networks.

Key words: semantics, AI, cognition, brain, model
\end{abstract}

\section{INTRODUCTION}

In this article we attempted to model brain's knowledge storing and processing. We use modern discoveries from the cognitive science, natural language processing and generally with the artificial intelligence and try to understand the neural basis of language and thought.

Knowledge can be stored in different ways. One possibility is to use semantic networks. Similar to proposed method is also Conceptual Dependency (CD) theory introduced by Roger C. Schank, because Schank's method also stores knowledge to the very universal network and this network also allows to store relation between objects and events in chronological sequence. More information about knowledge storing can be found in (Rich, 1983).

There are many theories of the neural representation of knowledge and models of a knowledge storing and processing in the human brain. An interesting way how to store knowledge is to store it in stories as they happened in an environment. A few of the most significant models which store stories had been implemented in the programs like MARGIE, SAM and PAM. These programs are based on the Conceptual Dependency (CD) theory by Roger C. Schank (Schank \& Tesler, 1969).

The programs SAM and PAM successfully showed how knowledge storing is important, but both programs were unable to process data out of their domain. SAM had difficulties with recognition plans and PAM had problems with understanding simple stories with events but without aims.

Vilem Benes introduces in (Benes, 2011) the idea that: "Till now, most AI solutions are narrow, limited only to some given domain. Designing AI without the aim for generality often encourages using domain-dependent tricks and knowledge (Goertzel, 2008) and leads to solutions that are not robust and adaptive - and dumb."

We also decided to create an AGI agent controlled by our proposed method. Our method is oriented to process: story events, environment objects and text information.

\section{METHODS}

A semantic network is a network which represents semantic relations among concepts. It is a directed or undirected graph consisting of vertexes, which represent concepts, and edges. Semantic networks are often used for the knowledge representation. Semantic networks only provide a structure into which nodes representing information can be placed.
Roger C. Schank found a suitable Conceptual Dependency model of decoding scripts into diagrams. This diagrams are still too complicated and therefore far from network in a human brain. Model with a simpler network has been developed. Description of CD can be found in (Schank \& Tesler, 1969) and (Schank, 1975). CD representation provides both a structure and a specific set of primitives out of which representations of particular pieces of information can be constructed. Proposed metod is similar to CD in many ways.

Proposed method consists of: environment (e. g. 2D virtual world), network (based on semantic neural network), interface (it is between the environment and the network), storing the knowledge and processing the stored knowledge.

A new algorithm which will be able to complete stories will be implemented. There will be possible to mark any event as predictable. Before the program reads and processes these events, the algorithm will try to guess them. We use the statistics to define how many marked events were successfully guessed by the program and this is the way how the program can be benchmarked.

Main idea of the algorithm is spreading activation applied on semantic neural network. This means copying activation potential from one node to connected ones as its in semantic neural network described in (Dudar \& Shuklin, 2000).

It will be also based on activation signal propagation direction changes. In odd iterations there will be used edge direction propagation there and we use opposite direction in even iterations. The exact algorithm is under development now and it will be described in one of our future papers.

There are two expected benefits of our method. First benefit is universality - we developed a generic network able to store any kind of knowledge (correlation with the human brain). However, it is necessary to implement input and output network interface for each of the new kind of information. The second benefit is a similarity with human brain: neurons are represented by nodes, and synapses are represented by edges with weights.

Possible disadvantage and limitation of our method is that it supports only story oriented environments.

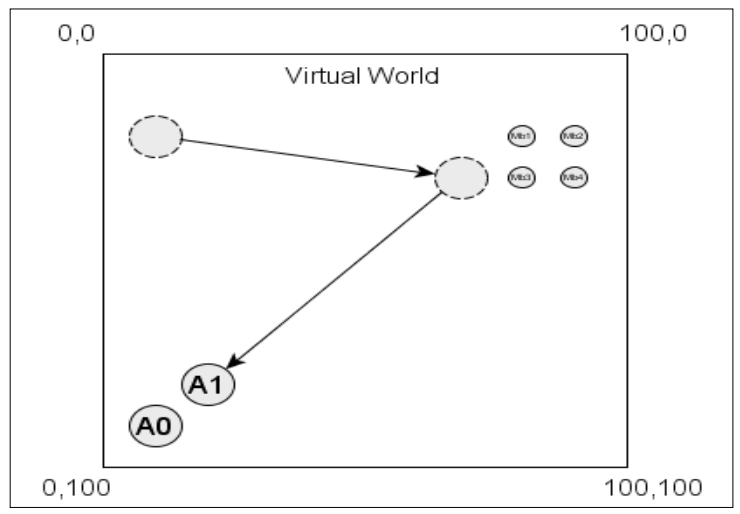

Fig. 1. Virtual world - a story of agent A1 


\section{PROPOSED NETWORK}

Because it is difficult to define words abstractly, we need a real world or better a simple virtual world with the objects.

At this phase of development there is a simple 2D grid virtual world enough. Objects in this world are agents and lifeless objects.

A lifeless object is e. g. eatable mushroom. Green and blue ones increase health and red ones decrease health. Agents health decreases continuously in the time, so agents are forced to search good mushrooms and when count of good mushrooms is insufficient, agents need to cooperate and to communicate. The text communication is the only form how agents can interact. And this is the main point of agents chatting in virtual world. More information about virtual worlds can be found in (Marik et al., 2007).

This virtual world is also similar to Capture region environment described in (Benes, 2011). See Fig. 1. There are two agents and four "food" units represented as mushrooms in this virtual world. Agent "A1" visited the mushrooms and went to told agent "A0" the fact that there are the mushrooms there. Nevertheless, main feature of the proposed method is generality. It is possible to add new types of objects or to change whole environment wish no changes to the network or to the algorithm processing stored knowledge.

However, proposed network has edges without any semantic information which makes it more dynamic, universal and closer to the real brain. How this information will be stored in the proposed network). Semantic meaning of nodes is defined by connected ones. There are two types of nodes: created nodes in learning process and predefined ones. The predefined ones are basic nodes defining meaning of other nodes. The predefined nodes are atomic nodes, e. g. quantity or count (numbers $1,2,3, \ldots$ ), type of interaction (see, message, ...) and direction (from, to, ...). Demonstration of these predefined nodes and how are used to define events in stories is on Fig. 2.

These predefined nodes represent concrete or abstract objects, events, etc and these objects can be in different types of environment, e. g. 2D virtual world, 3D virtual world or chessboard. Knowledge stored in the network is stored in the story trees. These stories are built by processing events received from environment.

Assume existing virtual world containing virtual rooms in 2D grid and virtual persons called agents. Suppose the agents can move one room in horizontal and vertical direction in each step. Let there is an event is the story that agent B came to room in which is agent A already. This event will be added to the network as it was shown on Fig. 2 introduced above. There are special predefined nodes in rectangle "Predefined special nodes". Each node in rectangle "Nodes created in learning process" except node "story \#123" is added when the event is added. Node labels on this figure are only as information for user or this article reader and algorithms don't have access to them.

Demonstration of proposed model on Fig. 2 shows a simple story with one event "agent B came to agent A". This story is stored in the semantic network. There is a part of semantic network containing node representing current story in big rectangle on the right side. The rectangle also contains nodes which directly define the story. These nodes in more complex stories will be possible to visually expand by the user to rectangle of defining nodes. After this expansion a new rectangle will be shown. Each network is divided into areas. The meanings of these areas are predefined and respond to predefined nodes categories. These areas are: numbers, directions, speeds of change, directions, etc. It will be possible to add to the application any kind of predefined nodes.

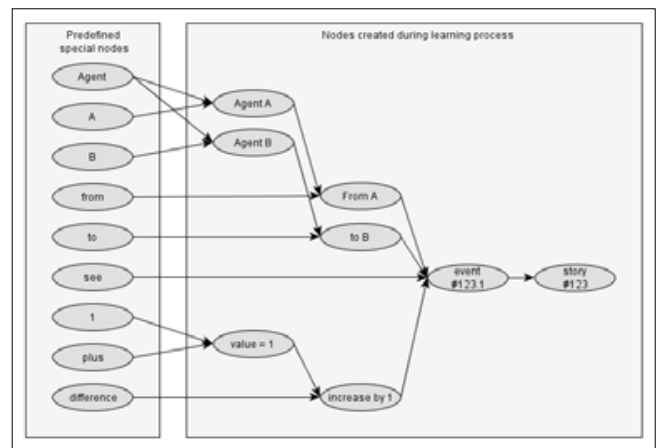

Fig. 2. A story with only one event "agent B came to agent A"

\section{FUTURE RESEARCH}

In the near future we are finishing the story editor. The program is under development at this time and it is already partially completed.

The program should demonstrate handling at least with functions like: basic story completing, logics, social understanding, grammar, knowledge transmission between agents. There are more functions in future plans, e. g. fuzzy logic and trustfulness.

\section{CONCLUSION}

A new method which allows knowledge storing into semantic neural network has been proposed here. Structure of this network tries to model a structure of the real human brain. Nodes and edges are significantly closer to real neurons and synapses than models in other methods. Proposed method is partially implemented and tested at this time. There will be user defined stories with some events marked as "to guess" by the algorithm based on spreading activation. The algorithm will be optimized to be able to "guess" the most of test events. This means that the controlled agent will almost have a human behavior if the tested stories will be from the real human world.

\section{REFERENCES}

Benes, V. (2011). Reactive Layer in AGI Agent: Implementation of Adaptive Reactive Behavior and Beyond (BAGIB agent). In: Proceedings of ICAART 2011 the International Conference on Agents and Artificial Intelligence, Vol. 1. Rome, Italy

Dudar Z.V. \& Shuklin D.E. (2000). Semantic neural net as a formal Neumann, J., 1966 language for texts description and parsing in natural language, In: Radioelectronika $i$ informatika KhTURE, no 3, pp. 72-76, ISSN: 1563-0064, Kharkov, Ukraine.

Goertzel, B. (2008). AI and AGI: Past, Present and Future, AGI-08 Conference. University of Memphis, online: ttp://www.acceleratingfuture.com/peopleblog/? p=1814

Marik, V.; Stepankova O. \& Lazansky J. (2007): Umela inteligence 5, Academia, Prague, Czech Republic

Rich E. (1983). Artificial intelligence, The University of Texas at Austin, NY, USA

Schank, C. \& Tesler, L. (1969). A conceptual dependency parser for natural language, Association for Computational Linguistics, Morristown, NJ, USA

Schank, C. (1975). The primitive acts of conceptual dependency, IN: TINLAP '75: Proceedings of the 1975 workshop on Theoretical issues in natural language processing, pp. 34-37, Association for Computational Linguistics, doi: 10.3115/980190.980205, Morristown, NJ, USA 